\title{
Dynamique d'éléments traces dans les précipitations sous le couvert de 2 pessières peu polluées de Suisse romande
}

\author{
O Atteia 1, É Dambrine 2 \\ 1 IATE-Pédologie, École polytechnique fédérale de Lausanne, Ecublens, 1015 Lausanne, Suisse ; \\ ${ }^{2}$ CFR-INRA, laboratoire des sols forestiers, 54280 Champenoux, France
}

(Reçu le 16 décembre 1992; accepté 26 février 1993)

\begin{abstract}
Résumé - Ce travail présente la composition des précipitations hors et sous couvert dans 2 pessières de Suisse romande situées dans des environnements très distincts. Le calcul de la différence entre les flux d'éléments déposés sous couvert et hors couvert permet de séparer les éléments dont les flux augmentent fortement lors de leur traversée des houppiers (Mn et Rb), des éléments légèrement enrichis ( $\mathrm{Sr}$ et $\mathrm{Ba}$ ), des éléments peu influencés par ce phénomène $(\mathrm{V}, \mathrm{Cr}, \mathrm{Ni}, \mathrm{Zn}$ et $\mathrm{Pb}$ ) et des éléments retenus par les aiguilles ( $B$ et $\mathrm{Fe}$ ). L'analyse de la structure chimique des solutions montre que le passage dans les houppiers induit de très nettes corrélations entre les éléments dans les solutions. Cet effet est attribué aux apports acidifiants et à l'équilibre chimique des solutions à la surface des aiguilles. Il est donc difficile de différencier la part des apports par les dépôts secs et par la récrétion grâce à cette technique. Néanmoins, sur ces sites peu pollués, il est clair que les concentrations de la plupart des métaux lourds dans les pluviolessivats sont peu influencées par les dépôts secs. Ceci est attribué à la faible pollution des sites. À l'inverse l'enrichissement en Rb est principalement dû au recyclage biologique et les concentrations de $\mathrm{Mn}$ et $\mathrm{Ba}$ sont fortement influencées par ce phénomène. $\mathrm{Sr}$, comme $\mathrm{Ca}$, semble être concentré par les deux phénomènes : dépôts secs et récrétion. Nous avons constaté la rétention de $\mathrm{B}$ et $\mathrm{Fe}$ sur les houppiers.
\end{abstract}

traces / éléments minéraux / précipitation / pluviolessivats / dépôt atmosphérique / épicea / Suisse

Summary - Trace element dynamics in throughfall of 2 spruce forests of French Switzerland, with low pollution levels. The chemical composition of rainfall and throughfall of 2 sites located in very different environments in Switzerland are compared. Calculation of the flux ratios from rainfall and throughfall leads to a differentiation between elements which are highly concentrated when passing though the tree crowns ( $\mathrm{Mn}$ and $\mathrm{Rb}$ ), slightly enriched elements ( $\mathrm{Sr}$ and $\mathrm{Ba}$ ), elements unaffected by this phenomenom ( $\mathrm{V}, \mathrm{Cr}, \mathrm{Ni}, \mathrm{Zn}$ and $\mathrm{Pb}$ ) and element retained by the needles ( $B$ and $\mathrm{Fe}$ ). Analysis of the multielemental chemical structure of solution shows that tree crown induces clear correlations between element concentrations. This effect is attributed to the input of acidifying anions and to the existence of a chemical equilibrium on the needle surface. It is therefore difficult to differentiate between dry deposition input and recretion. Nevertheless it is evident that the concen- 
trations of most of the heavy metals are not influenced by dry deposition. This could be due to the low pollution level at these sites. Conversely, Rb enrichment is mainly due to biological cycling and $\mathrm{Mn}$ and Ba concentrations are highly modified by this phenomenom. $\mathrm{Sr}$, like $\mathrm{Ca}$, seems to be enriched by 2 different phenomena: dry deposition and recretion. We found some retention of $F e$ and $B$ on the tree crowns.

trace elements / mineral elements / precipitation / throughfall / atmospheric deposition / spruce / Switzerland

\section{INTRODUCTION}

De nombreuses études portant sur le dépôt d'éléments minéraux hors et sous couvert forestier ont été développées au cours de la dernière décennie, dans le but de quantifier l'importance de ces apports, sur le plan nutritif (Ellis et al, 1983) comme sur celui de la pollution (Canter, 1986). Dans ces deux domaines, il est apparu que ces apports variaient considérablement sur le plan spatial (Kostelnik et al, 1989) et que leur importance était souvent primordiale pour l'évolution de la fertilité des sols forestiers (Marschner, 1986). Cependant, d'une part, la quantification de ces apports s'est avérée difficile, tout particulièrement pour ceux déposés sous forme occulte, et, d'autre part, les investigations ont essentiellement porté sur les éléments majeurs et peu sur les éléments traces. Si les dépôts humides présentent peu de difficultés de mesure, les dépôts secs ou occultes sont difficiles à quantifier car on ne dispose pas de capteurs présentant les caractères aérodynamiques et les propriétés de surface d'un couvert forestier (lbrahim et al, 1983). Par ailleurs la modélisation de ces dépôts nécessite la mesure de nombreux paramètres, dont les valeurs sont variables en fonction du site d'étude (Greenfelt et al, 1978). La difficulté a été contournée par plusieurs auteurs qui ont utilisé les flux d'éléments sous couvert comme indicateur du dépôt atmosphérique total. En fait ce flux est la résultante de plusieurs phénomènes.

i) Dépôt humide. Une partie des précipitations, temporairement stockée sur les aiguilles ou les feuilles, s'évapore ; ce phénomène est l'interception. La pluie suivante sera ainsi enrichie des éléments minéraux restés sur le feuillage (Veen et Dolman, 1989). L'interception a donc une action directe sur les teneurs en éléments minéraux des précipitaticns sous couvert par concentration relative. Elle ne modifie pas les flux d'éléments déposés.

ii) Lessivage par les précipitations humides de dépôts secs ou occultes accumulés à la surface des feuilles (Garland, 1979 ; Ivens et al, 1989). La surface et la rugosité du couvert forestier entraînent l'impaction et l'adsorption sur les feuilles de substances présentes dans l'air sous forme d'aérosol, de particule ou de gaz. Sur les sites pollués, ces dépôts peuvent composer la majorité du dépôt total (Godt et al, 1986).

iii) Absorption d'éléments d'origine atmosphérique par les arbres (Hoffmann et al, 1980). Les possibilités d'absorption dépendent de l'élément et des besoins de la plante en l'élément considéré (Swank, 1984).

iv) Rétention physique de particules, ou rétention chimique d'ions par les cires cuticulaires ; ce phénomène est mal connu jusqu'à présent (Grosch, 1986).

v) Lessivage de produits récrétés par les houppiers naturellement ou sous l'action des dépôts atmosphériques (Tukey et Witt- 
ner, 1957). Ce lessivage est soumis aux équilibres chimiques advenant dans la fine couche d'eau présente à la surface des aiguilles (Ulrich, 1983 ; Cronan et Reiners, 1983).

La contribution relative de chacun de ces phénomènes au dépôt sous couvert est approximativement connue pour certains éléments. Ainsi $\mathrm{Na}, \mathrm{Cl}, \mathrm{SO}_{4}$ et $\mathrm{NO}_{3}$ ne sont pas absorbés par l'arbre, ni retenus à la surface des feuilles, ni récrétés (Lindberg et al, 1982 ; Garten et al, 1988), de sorte que le flux sous couvert totalise le dépôt humide et les dépôts secs et occultes. À l'inverse $\mathrm{K}$ est intensément récrété tandis que les métaux lourds comme $\mathrm{Pb}, \mathrm{Cu}$ ou $\mathrm{Cr}$ s'accumulent à la surface des feuilles. L'acidité de la solution au contact des feuilles favorise la récrétion de $\mathrm{Ca}$ et $\mathrm{Mg}$ (Horntvedt et al, 1980), cependant des dépôts secs substantiels de ces éléments ont été mesurés ou calculés (Lindberg et al, 1982 ; Bredemeier, 1988). Ces travaux ont principalement concerné les éléments majeurs, le comportement des éléments traces a été très peu étudié excepté celui des métaux lourds comme $\mathrm{Ni}, \mathrm{Zn}, \mathrm{Pb}, \mathrm{Cu}$ et $\mathrm{Cd}$ (Heinrichs et Mayer, 1980), largement émis dans l'atmosphère par de nombreuses activités humaines.

Cet article présente les dynamiques d'éléments traces en relation avec celles des éléments majeurs, lors de la traversée des précipitations à travers les houppiers. Sont considérés comme éléments traces ceux dont les concentrations moyennes sont généralement inférieures à $100 \mu \mathrm{g} / \mathrm{l}$. Parmi ceux ci : (i) des métaux dont la concentration varie fortement en fonction de l'environnement: $\mathrm{Mn}$ et $\mathrm{Fe}$; (ii) des métaux lourds : V, Cr, Ni, Cu, $\mathrm{Zn}$ et $\mathrm{Pb}$; (iii) des alcalins et alcalino-terreux : $\mathrm{Rb}, \mathrm{Sr}$ et $\mathrm{Ba}$; et (iiii) un métalloïde : $\mathrm{B}$. Les éléments (terres rares, autres métaux lourds) dont les concentrations sont inférieures à $1 \mu \mathrm{g} / \mathrm{l}$ dans les solutions analysées n'ont pas été étudiés.

\section{MATÉRIEL ET MÉTHODES}

\section{Site (fig 1)}

\section{Le site de "Lutry"}

Il est situé à $15 \mathrm{~km}$ au nord de Lausanne à une altitude de $900 \mathrm{~m}$. Le substrat géologique est constitué de molasse burdigalienne, couverture fortement représentée sur le plateau suisse. Le sol est un sol brun acide (Dystric Cambisol) dont le $\mathrm{pH}$ eau est de 3,8 en surface, de 4,5 entre 10 $\mathrm{cm}$ et $1,4 \mathrm{~m}$ de profondeur, le front de décarbonatation est atteint à $2,5 \mathrm{~m}$. La pluviométrie annuelle moyenne est de $1250 \mathrm{~mm}$. Des traces discrètes (concrétions) d'hydromorphie témoignent de la remontée hivernale de la nappe jusqu'à la surface du sol (Atteia, 1992). II s'agit d'une plantation d'épicéa de 80 à 100 ans, d'environ $35 \mathrm{~m}$ de hauteur dominante. Sa productivité est de 10 à $14 \mathrm{~m}^{3} / \mathrm{ha}$. Le sous-bois est occupé par des ronces (Rubus sp). Le peuplement se trouve dans une zone d'élevage et d'exploitation forestière, les activités agricoles étant peu intensives.

\section{Le site "Jura"}

II est situé à $1360 \mathrm{~m}$ d'altitude près du mont Tendre (Suisse), loin de toute zone d'habitation. La roche sous-jacente est un calcaire dur du Jurassique (Kimmeridgien). Le sol est un sol brun calcique peu profond ( 20 à $25 \mathrm{~cm}$ ) L'horizon de contact entre sol et roche-mère est constitué en majorité de cailloux calcaires de taille centimétrique. Ce site est proche de la limite altitudinale de la forêt dans le Jura. Aussi, cette forêt mixte, composée de hêtres, de sapins et d'épicéas, présente une croissance très lente, la productivité annuelle ne dépassant pas $4 \mathrm{~m}^{3} / \mathrm{ha}$. La forêt n'est pas exploitée, le sous-bois est formé de quelques rares buissons. $\dot{A}$ proximité se trouvent des pâtures estivales extensivement parcourues par des bovins. Le site sous couvert se trouve sous un bouquet d'épicéa d'une vingtaine de mètres de haut en moyenne.

\section{Matériel de prélèvement}

Hors couvert, un collecteur par site, formé par un entonnoir en polyéthylène de $30 \mathrm{~cm}^{2}$, est 
placé dans une clairière à proximité immédiate du site sous couvert. Sous le couvert forestier, le système de prélèvement est constitué de trois gouttières hémicylindriques en polyéthylène, de $2 \mathrm{~m}$ de long et $10 \mathrm{~cm}$ de large, disposées en éventail. La disposition des gouttières est effectuée de manière à refléter approximativement la proportion de trouées dans le couvert. Dans le site Jura, les collecteurs ont été placés exclusivement sous un couvert d'épicéa. Une faible pente permet l'évacuation des eaux vers un récipient. L'utilisation de gouttières permet une bonne intégration de la variabilité spatiale quantitative et qualitative des pluviolessivats (Kostelnik et al, 1989).

Les 2 types de collecteurs sont reliés à un même type de dispositif de stockage des solutions (réservoir de $30 \mathrm{I}$ en polypropylène enterré dans le sol) par un tuyau en silicone. Tous les matériaux utilisés ont subi des tests d'inertie chimique et ont été rincés à l'acide nitrique $10 \%$ et à l'eau déminéralisée avant installation.

\section{Période d'échantillonnage}

L'intervalle d'échantillonnage est déterminé en fonction de l'estimation des quantités de pluies reçues sur le site, il peut ainsi varier d'une semaine à un mois.

Sur le site du Jura, nous disposons de 22 campagnes de mesure comprenant des échantillons de pluie et de pluviolessivat, sur une période de 20 mois (du 15 février 1990 au 15 octobre 1991). Sur le site de Lutry, nous disposons de 30 campagnes sur la même période pour les pluviolessivats et de $12 \mathrm{cam}$ pagnes étalées sur 7 mois depuis le $1^{\text {er }}$ mars 1991 pour les pluies. Ce facteur est pris en compte dans l'exploitation des résultats.

\section{Techniques analytiques}

Après le prélèvement, les échantillons sont filtrés $(0,45 \mu \mathrm{m})$ puis acidifiés avec de l'acide nitrique suprapur $0,2 \%$ et conservés à $+4^{\circ} \mathrm{C}$ en vue de l'analyse des cations. Les échantillons sont congelés directement après filtration en vue de l'analyse des anions.

Les éléments traces, ainsi que certains éléments majeurs ( $\mathrm{Ca}, \mathrm{Al}, \mathrm{B}, \mathrm{V}, \mathrm{Cr}, \mathrm{Ni}, \mathrm{Co}, \mathrm{Fe}, \mathrm{Mn}$, $\mathrm{Cu}, \mathrm{Zn}, \mathrm{Rb}, \mathrm{Sr}, \mathrm{Ba}, \mathrm{Pb}$ ), sont analysés par une ICP-MS (inductively coupled plasma-mass spec-

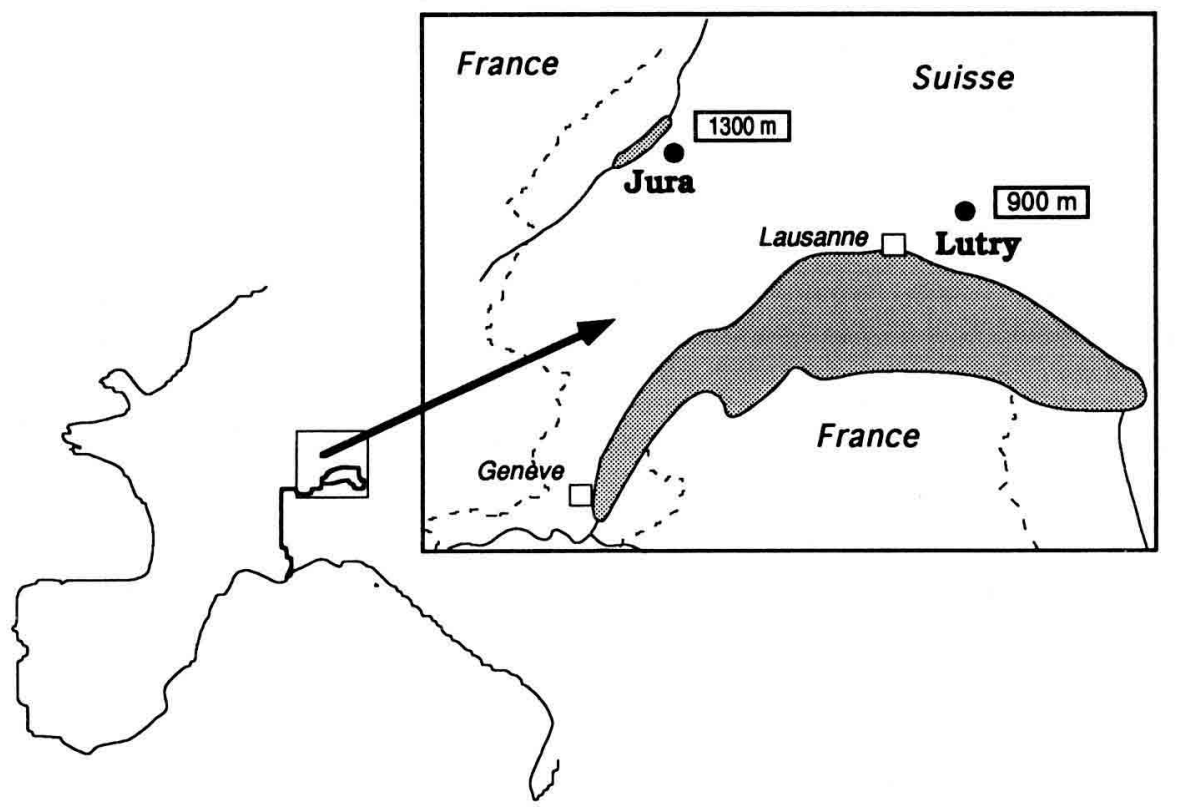

Fig 1. Localisation des sites. 
trometer, VG Plasmaquad), en utilisant I'Yttrium comme standard interne. Dans un but comparatif, les éléments majeurs $(\mathrm{Ca}, \mathrm{Si}, \mathrm{Mg}, \mathrm{K}, \mathrm{Na}, \mathrm{Al})$ sont analysés avec un DCP-AES (ARL Spectraspan II) en présence de $2 \%$ de $\mathrm{LiCl}$ $(500 \mathrm{ppm})$ afin de minimiser les différences d'absorption entre alcalins et alcalino-terreux. Une partie des analyses de sulfates (sous forme S) a été réalisée en ICP (Jobin-Yvon 38+), une autre partie a été effectuée en chromatographie ionique avec suppresseur (Sykam); les résultats montrent une bonne concordance, avec une erreur inférieure à $10 \%$. Les anions $\left(\mathrm{NO}_{3}, \mathrm{NH}_{4}\right.$, $\mathrm{Cl}$ ) sont analysés par colorimétrie automatique (Autoanalyser Technicon). La précision des analyses, établie par des comparaisons interlaboratoires ou inter-séries pour certains éléments, est inférieure ou égale à $10 \%$ pour la plupart des éléments ; seule l'erreur sur Fe est supérieure à $10 \%$ (de l'ordre de 20 à $30 \%$ ). Pour les éléments dont les concentrations sont inférieures à 2 ou $3 \mu \mathrm{g} / \mathrm{l}(\mathrm{Co}, \mathrm{Cr}, \mathrm{V})$, l'erreur absolue est intérieure à $1 \mu \mathrm{g} / \mathrm{l}$ (Atteia, 1992).

\section{RÉSULTATS}

\section{Apports d'éléments hors couvert}

Les flux annuels d'éléments minéraux hors couvert sur les deux sites sont présentés dans le tableau I. Les valeurs obtenues concernant les éléments traces sont faibles puisque seuls les flux moyens de $\mathrm{Zn}$ dépassent $100 \mathrm{~g} / \mathrm{ha} / \mathrm{an}$ sur les deux sites. Nous avons comparé les flux de $V$, $\mathrm{Mn}, \mathrm{Ni}, \mathrm{Cu}, \mathrm{Zn}$ et $\mathrm{Pb}$ mesurés sur un réseau étendu couvrant la majorité de la Suisse romande aux flux cités dans les revues bibliographiques de Bergkvist et al (1989) et Galloway et al (1982) (tableau II) ; les données concernant les autres éléments étant rares. Cette analyse est développée sur une plus vaste échelle par

Tableau I. Flux annuels d'éléments majeurs et traces dans les précipitations hors couvert des sites "Jura" et "Lutry".

\begin{tabular}{lcccccccc}
\hline Kg/ha/an & $\mathrm{Si}$ & $\mathrm{Ca}$ & $\mathrm{Cl}$ & $\mathrm{Na}$ & $\mathrm{S}-\mathrm{SO}_{4}$ & $\mathrm{~N}-\mathrm{NO}_{3}$ & $\mathrm{Mg}$ & $K$ \\
\hline J hc 1 & 7.66 & 5.4 & 5.61 & 2.17 & 6.77 & 4.85 & 0.14 & 1.79 \\
J hc2 & 11.3 & 6.17 & 4.8 & 1.05 & 6.9 & 4.89 & 0.11 & 3.49 \\
L hc2 & 5.88 & 8.99 & 3.75 & 0.66 & 8.36 & 5.26 & 0.1 & 1.13 \\
\hline
\end{tabular}

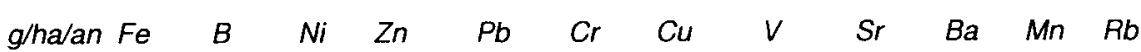

\begin{tabular}{lcccccccccccc}
\hline J hc1 & 69.6 & 110 & 3.47 & 362 & 62.6 & 9.53 & 59.7 & 4.52 & 6.67 & 15.5 & 55.2 & 6.89 \\
J hc2 & 75 & 56 & 5.98 & 346 & 67.9 & 16 & 84.5 & 5.7 & 18.8 & 14.6 & 68.1 & 10.9 \\
L hc2 & 112 & 125 & 7.96 & 242 & 26 & 1.18 & 26.2 & 5.65 & 25.2 & 24.7 & 129 & 1.94
\end{tabular}

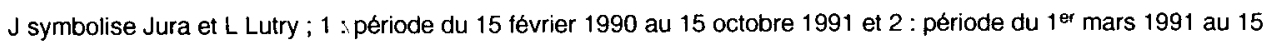
octobre 1991. 
Tableau II. Flux d'éléments minéraux dans les précipitations hors couvert comparées avec des données bibliographiques.

\begin{tabular}{lcccccccc}
\hline Kg/ha/an & $\begin{array}{c}\text { Suisse } \\
\text { romande }\end{array}$ & $\begin{array}{c}\text { France } \\
\text { Ouest }\end{array}$ & $\begin{array}{c}\text { France } \\
\text { Est }\end{array}$ & Autriche & $\begin{array}{c}\text { Allemagne } \\
\text { (Hesse) }\end{array}$ & Hollande & Finlande NE USA \\
\hline Réf. & & 1 & 1 & 2 & 3 & 4 & 5 & 6 \\
$\mathrm{~S}^{-S_{4}}$ & $4,6-8,6$ & $4,8-6,4$ & $18-24$ & $7-15$ & $12,3-21,9$ & $6-70$ & $3-10$ & $3,3-10$ \\
$\mathrm{~N}_{4} \mathrm{NO}_{3}$ & $4,4-7,5$ & $0,8-2,4$ & $3,8-6,2$ & $3-7$ & $4,5-7,7$ & $5,6-34$ & $1-3$ & \\
\hline
\end{tabular}

\begin{tabular}{|c|c|c|c|c|c|}
\hline g/ah/an & $\begin{array}{l}\text { Suisse } \\
\text { romande }\end{array}$ & $\begin{array}{l}\text { Sites } \\
\text { recules }\end{array}$ & $\begin{array}{c}\text { Sites } \\
\text { ruraux }\end{array}$ & Allemagne & Suède \\
\hline $\begin{array}{l}\text { Réf } \\
\mathrm{V} \\
\mathrm{Cr} \\
\mathrm{Mn} \\
\mathrm{Ni} \\
\mathrm{Cu} \\
\mathrm{Zn} \\
\mathrm{Pb}\end{array}$ & $\begin{array}{c}4-6,7 \\
0,1-9,5 \\
28-90 \\
0,1-8,4 \\
6-54 \\
77-406 \\
4,3-51,6\end{array}$ & $\begin{array}{c}1 \\
1 \\
0.8\end{array}$ & $\begin{array}{c}7 \\
10-500 \\
10-360 \\
20-250 \\
10-500 \\
20-230 \\
40-820 \\
19-246\end{array}$ & $\begin{array}{c}8 \\
1,8-6 \\
4,5-42 \\
11-86 \\
90-410 \\
60-390\end{array}$ & $\begin{array}{c}8 \\
1,3-8 \\
\\
1,8-12 \\
7-40 \\
100-318 \\
60-150\end{array}$ \\
\hline
\end{tabular}

Flux (kg/ha/an pour les majeurs et g/ha/an pour les traces) minimum et maximum sur les sites de "Suisse romande" étudiés, comparés à des valeurs provenant d'autres pays ; références : 1: Hennequin et al 1984, 2: Smidt, 1988, 3: Bechtel et al, 1986, 4: Bresser, 1990, 5: Järvinen, 1986, 6: Summers et al, 1986, 7: Galloway et al, 1982, 8: Bergkvist et al, 1989

ailleurs (Atteia, 1993) mais les principales conclusions sont les suivantes : les flux de $\mathrm{V}, \mathrm{Mn}, \mathrm{Ni}$ et $\mathrm{Pb}$ sont inférieurs à ceux cités pour les sites ruraux allemands et pour les sites peu pollués du nord de la Suède. Par contre, les flux de $\mathrm{Cu}$ et $\mathrm{Zn}$ sont semblables à ceux cités par Bergkvist (1989) pour différents lieux de Suède. En ce qui concerne les éléments majeurs, les dépôts hors couvert de $\mathrm{S}-\mathrm{SO}_{4}$ et $\mathrm{N}-\mathrm{NO}_{3}$ sont faibles dans le Jura, respectivement 6 et 4 $\mathrm{kg} / \mathrm{ha} / \mathrm{an}$, tandis que ceux mesurés à Lutry se situent à un niveau plus élevé $(8,4$ et $5,3 \mathrm{~kg} / \mathrm{ha} / \mathrm{an}$ ) correspondant aux flux moyens mesurés dans les Vosges pendant la même période (Aschan et al, 1991). L'acidité moyenne des précipitations hors couvert est faible $(\mathrm{pH} 4,5$ à Lutry et 5 dans le Jura). On note de même des flux de Si déposés hors couvert relativement élevés (supérieurs à $6 \mathrm{~kg} / \mathrm{ha} / \mathrm{an}$ ), probablement attribuables à des particules terrigènes. Nous sommes donc en présence de sites peu pollués dans le contexte européen.

\section{Structure chimique des précipitations hors et sous couvert}

Nous avons effectué des analyses en composantes principales (tableau III) afin de déterminer les structures chimiques des précipitations hors couvert, d'une part, et sous couvert, d'autre part (tous sites confondus). Sous couvert, le premier facteur explique $71 \%$ de la variance et trois facteurs suffisent pour expliquer $92 \%$ de la 
variance, alors que, hors couvert, il faut 6 facteurs pour expliquer $90 \%$ de la variance. Les projections des éléments sur les axes factoriels sont plus dispersées hors couvert que sous couvert. Hors couvert, 3 éléments sont nettement reliés aux axes principaux, alors que ce nombre est de 16 sous couvert. Ainsi, le passage à travers le couvert forestier structure nettement la composition des solutions. Cette analyse conduit à différencier 3 groupes quasiment indépendants : (i) $\mathrm{Rb}-\mathrm{K}$, (ii) $\mathrm{Na}$ et $\mathrm{Cl}$ et enfin (iii) $\mathrm{Sr}, \mathrm{Zn}, \mathrm{Ni}, \mathrm{Pb}, \mathrm{SO}_{4}, \mathrm{Ca}$ et $\mathrm{NO}_{3}$. Pour compléter cette analyse, nous avons calculé les coefficients de corrélation entre les concentrations hors couvert et sous couvert pour tous les éléments.
Les coefficients de corrélations sont tous très faibles, mis à part ceux qui concernent $\mathrm{Na}$ et $\mathrm{Cl}$.

\section{Modification des flux d'éléments lors du passage sous couvert}

L'interception, suivie de l'évaporation d'une fraction des précipitations retenues sur le couvert induit une élévation des concentrations dans les précipitations sous couvert. Afin d'éliminer cet effet, nous raisonnons en terme de flux d'élément, l'interception n'influençant pas ces flux. Les périodes de mesure n'ayant pas la même longueur sur

Tableau III. Analyse en composante principale sur la composition totale des solutions.

Pluies

\begin{tabular}{lccccc}
\hline & & & & & \\
& & 2 & 3 & 4 & 5 \\
\hline \% var & 33.6 & 24.1 & 11.8 & 10.0 & 9.1 \\
\% vcum & 33.6 & 57.6 & 69.5 & 79.5 & 88.6 \\
$\mathrm{Ca}$ & 0.17 & 0.64 & -0.44 & 0.38 & 0.41 \\
$\mathrm{Si}$ & 0.57 & -0.53 & 0.04 & 0.49 & 0.04 \\
$\mathrm{Mg}$ & -0.57 & 0.06 & 0.61 & 0.05 & 0.35 \\
$\mathrm{~K}$ & -0.23 & -0.33 & 0.66 & 0.31 & 0.48 \\
$\mathrm{CL}$ & 0.82 & 0.23 & 0.21 & 0.36 & -0.03 \\
$\mathrm{NO} 3$ & 0.49 & -0.14 & -0.6 & -0.42 & 0.29 \\
$\mathrm{Na}$ & 0.51 & -0.59 & -0.06 & -0.32 & 0.43 \\
$\mathrm{SO} 4$ & 0.25 & 0.48 & -0.72 & 0.36 & 0.23 \\
$\mathrm{~B}$ & 0.66 & -0.56 & -0.1 & 0.31 & -0.21 \\
$\mathrm{~V}$ & 0.83 & 0.04 & 0.14 & 0.4 & -0.02 \\
$\mathrm{Cr}$ & 0.23 & -0.60 & -0.13 & 0.27 & -0.33 \\
$\mathrm{Fe}$ & 0.74 & 0.04 & 0.19 & -0.56 & 0.27 \\
$\mathrm{Mn}$ & 0.73 & 0.61 & 0.19 & -0.03 & -0.13 \\
$\mathrm{Ni}$ & 0.76 & 0.49 & 0.26 & -0.16 & -0.18 \\
$\mathrm{Cu}$ & 0.52 & -0.66 & -0.13 & -0.02 & -0.29 \\
$\mathrm{Zn}$ & 0.77 & 0.43 & 0.37 & 0.07 & -0.23 \\
$\mathrm{Rb}$ & 0.36 & -0.55 & 0.14 & 0.26 & 0.59 \\
$\mathrm{Sr}$ & 0.14 & 0.83 & -0.07 & 0.14 & 0.38 \\
$\mathrm{Ba}$ & 0.68 & 0.41 & 0.27 & -0.36 & -0.02 \\
$\mathrm{~Pb}$ & 0.66 & -0.54 & -0.1 & -0.33 & 0.25 \\
& & & & & \\
\hline
\end{tabular}

Pluvio-lessivats

\begin{tabular}{ccccc}
\hline 1 & 2 & 3 & 4 & 5 \\
\hline $\mathbf{7 1 . 0}$ & 11.6 & 9.5 & 3.8 & 2.2 \\
$\mathbf{7 1}$ & $\mathbf{8 2 . 5}$ & $\mathbf{9 2}$ & $\mathbf{9 5 . 9}$ & $\mathbf{9 8}$ \\
$\mathbf{0 . 9 3}$ & -0.19 & -0.02 & 0.26 & 0.19 \\
$\mathbf{0 . 8 9}$ & 0.18 & 0.4 & 0.11 & 0.03 \\
$\mathbf{0 . 8 5}$ & -0.43 & 0.12 & 0.26 & 0.02 \\
$\mathbf{0 . 9 1}$ & -0.26 & 0.3 & -0.14 & -0.07 \\
$\mathbf{0 . 9 3}$ & 0.05 & -0.16 & -0.28 & 0.09 \\
$\mathbf{0 . 9 7}$ & 0.23 & 0.03 & -0.04 & 0.02 \\
$\mathbf{0 . 6 3}$ & -0.63 & 0.23 & -0.21 & -0.31 \\
$\mathbf{0 . 9 1}$ & 0.32 & 0.12 & 0.17 & 0.02 \\
$\mathbf{0 . 9 3}$ & 0.19 & 0.28 & -0.03 & 0.08 \\
$\mathbf{0 . 9 2}$ & 0.34 & 0.03 & 0.02 & -0.1 \\
$\mathbf{0 . 9 3}$ & 0.16 & -0.16 & 0.08 & 0.05 \\
$\mathbf{0 . 8 9}$ & 0.17 & 0.27 & $\mathbf{0 . 2 3}$ & -0.16 \\
$\mathbf{0 . 7 4}$ & 0.12 & -0.59 & -0.29 & 0.11 \\
$\mathbf{0 . 8 0}$ & -0.42 & -0.3 & -0.27 & 0.05 \\
$\mathbf{0 . 9 4}$ & 0.17 & 0.21 & -0.12 & -0.09 \\
$\mathbf{0 . 7 9}$ & 0.03 & -0.59 & $\mathbf{0 . 0 5}$ & -0.02 \\
$\mathbf{0 . 9 0}$ & -0.25 & 0.23 & -0.23 & -0.03 \\
$\mathbf{0 . 9 2}$ & -0.16 & -0.12 & 0.17 & 0.27 \\
-0.21 & -0.94 & 0.12 & $\mathbf{0 . 1 4}$ & 0.18 \\
$\mathbf{0 . 4 9}$ & -0.23 & -0.67 & $\mathbf{0 . 3 4}$ & -0.34 \\
\hline & & & & \\
\hline
\end{tabular}

Pour chaque facteur de l'ACP sont figurés le pourcentage de la variance expliquée par ce facteur (\% var), le pourcentage cumulé (\% vcum) et les corrélations entre les éléments et les facteurs ; si cette valeur est supérieure à 0,8 , elle est représentée en caractères gras. 
Tableau IV. Flux annuels d'éléments majeurs et traces dans les précipitations sous couvert des sites "Jura" et "Lutry".

\begin{tabular}{lcccccccc}
\hline Kg/ha/an & \multirow{2}{*}{$\mathrm{Si}$} & $\mathrm{Ca}$ & $\mathrm{Cl}$ & $\mathrm{Na}$ & $\mathrm{S}-\mathrm{SO}_{4}$ & $\mathrm{~N}-\mathrm{NO}_{3}$ & $\mathrm{Mg}$ & $K$ \\
\hline J sc1 & 0,7 & 8,77 & 10,2 & 3,41 & 7,84 & 8,16 & 0,96 & 11,8 \\
L sc1 & 0,52 & 10,3 & 8,38 & 2,08 & 14 & 10,3 & 1,27 & 8,45 \\
J sc2 & 1,18 & 9,32 & 4,73 & 1,5 & 22,3 & 8,19 & 1,05 & 11,6 \\
L sc2 & 2,2 & 14,4 & 9,82 & 1,57 & 16,7 & 18,3 & 1,69 & 17,4 \\
R J1 & 0,09 & 1,62 & 1,82 & 1,57 & 1,16 & 1,68 & 6,91 & 6,64 \\
R J2 & 0,11 & 1,51 & 0,98 & 1,43 & 3,24 & 1,67 & 9,33 & 3,34 \\
R L2 & 0,37 & 1,6 & 2,62 & 2,39 & 2 & 3,48 & 16,7 & 15,4 \\
\hline
\end{tabular}

\begin{tabular}{lcccccccccccc}
\hline g/ha/an $F e$ & $B$ & $N i$ & $Z n$ & $P b$ & Cr & $C u$ & $V$ & $S r$ & $B a$ & $M n$ & $R b$ \\
\hline J sc1 & 35,3 & 72,1 & 3,73 & 162 & 15,7 & 1,07 & 13,1 & 4,89 & 9,36 & 59,4 & 172 & 74,9 \\
L sc1 & 54,9 & 78,3 & 4,96 & 301 & 37,5 & 1,15 & 29,5 & 7,57 & 17,8 & 40,1 & 1192 & 48,5 \\
J sc2 & 18,6 & 56,9 & 4,73 & 119 & 14,2 & 0,3 & 14,4 & 3,49 & 21,1 & 48,7 & 172 & 65,1 \\
L sc2 & 115 & 104 & 4,9 & 247 & 33,6 & 1,59 & 32,4 & 12,7 & 34,3 & 52,9 & 1308 & 103 \\
R J1 & 0,51 & 065 & 1,08 & 0,45 & 0,25 & 0,11 & 0,22 & 1,08 & 1,4 & 3,84 & 3,12 & 10,9 \\
R J2 & 0,25 & 10,01 & 0,79 & 0,34 & 0,21 & 0,02 & 0,17 & 0,61 & 1,12 & 3,34 & 2,52 & 5,98 \\
R L2 & 1,03 & 0,83 & 0,62 & 1,02 & 1,29 & 1,34 & 1,24 & 2,25 & 1,36 & 2,14 & 10,1 & 52,7 \\
\end{tabular}

J signifie Jura et L Lutry ; SC symbolise "sous couvert" ; 1: periode du 15 février au 15 octobre $1991 ; 2:$ période du $1^{\text {er }}$ mars 1991 au 15 octobre 1991 ; R: rapport de flux sous couvert divisé par le flux hors couvert.

les 2 sites, nous présentons des moyennes mensuelles de flux calculées sur les périodes disponibes, mais, dans un but de comparaison, les données sont aussi présentées pour le Jura sur la période de prélèvement de 7 mois de Lutry.

Si l'on considère l'ensemble du feuillage comme une boîte noire, le rapport entre flux entrant et sortant définit une capacité de rétention ou de libération de chaque élément. Les valeurs des facteurs d'enrichissement (flux sortant / flux entrant) sont présentées dans le tableau IV. Mis à part $\mathrm{Ba}$ et $\mathrm{Ni}$, tous les éléments présentent, à Lutry, des rapports égaux ou supérieurs à ceux du "Jura". Le tableau IV présente également des flux moyens mensuels d'éléments minéraux enregistrés dans les deux stations. On observe une différence nette entre la station du Jura pour laquelle les flux d'éléments considérés comme acidifiants $\left(\mathrm{N}-\mathrm{NO}_{3}\right.$ et $\left.\mathrm{S}-\mathrm{SO}_{4}\right)$ restent faibles et Lutry où ils s'élèvent très nettement par rapport à ceux mesurés hors couvert (tableau II).

\section{Quatre groupes d'éléments se distinguent}

1. Les éléments fortement enrichis: $M n$, $\mathrm{Rb}$, ainsi que $\mathrm{Mg}$ et $\mathrm{K}$, dont les rapports entre flux sortant $(R)$ et flux entrant sont 
toujours supérieurs à 3 sur les deux sites. $\mathrm{Rb}$ et $\mathrm{Mn}$, quasiment absents dans les pluies, présentent les facteurs d'enrichissement les plus importants entre pluies et pluviolessivats. La corrélation entre $\mathrm{K}$ et $\mathrm{Rb}$ dans les pluviolessivats est forte (fig 2) et la droite de régression est la même sur ces 2 sites situés dans des environnements très différents.

Le cas de Mn est différent : les concentrations de cet élément ne sont pas corrélées à celles de $\mathrm{K}$ et $\mathrm{Rb}$ mais à celles de $\mathrm{SO}_{4}$. De plus, les droites de corrélation sont très différentes sur les deux sites (fig 2), la pente à Lutry étant beaucoup plus élevée qu'à "Jura".

2. Les éléments légèrement enrichis : $R$ variant de 1,2 à 3,8 selon les sites: $\mathrm{Sr}$ et $\mathrm{Ba}$. Les majeurs $\mathrm{Ca}, \mathrm{SO}_{4}, \mathrm{Cl}$ et $\mathrm{Na}$ se situent dans ce groupe. Les rapports d'enrichissement de Sr sont tout à fait semblables à ceux de $\mathrm{Ca}$. Les concentrations de ces deux éléments sont corrélées dans les pluies et dans les pluviolessivats (fig 3).

Ba est plus enrichi que $\mathrm{Ca}$ et $\mathrm{Sr}$ lors de son passage sous couvert. De plus, on remarque sur la figure 2 que les concentrations de cet élément dans les pluviolessivats sont plus fortes sur site calcaire que sur site acide.

3. Les éléments peu influencés par leur passage à travers le feuillage : $\mathrm{V}, \mathrm{Cr}, \mathrm{Ni}$, $\mathrm{Cu}, \mathrm{Zn}, \mathrm{Pb}$ (R proche de 1). Ce sont principalement des métaux lourds, les valeurs des facteurs d'enrichissement (R) pour ces éléments sont proches de 1 à Lutry et plus faibles sur Jura. La figure 4 présente l'évolution saisonnière des concentrations de certains de ces éléments dans les précipitations hors et sous le couvert à Jura. On remarque que les concentrations de $\mathrm{Cr}$, $\mathrm{Pb}, \mathrm{Cu}$ et $\mathrm{Zn}$ dans les pluies augmentent notablement vers la fin de l'hiver 19901991. Avant et après cette période, les concentrations dans les pluies et les pluviolessivats sont tout à fait semblables.
Lors de l'augmentation des concentrations, seules les concentrations de $\mathrm{Zn}$ augmentent dans les pluviolessivats alors que celles de $\mathrm{Cu}$ et $\mathrm{Pb}$ restent semblables aux valeurs précédentes. II s'agit donc d'une accumulation passagère de $\mathrm{Pb}$ et $\mathrm{Cu}$ sur le feuillage.

4. Les éléments retenus par le feuillage : $B$, $\mathrm{Fe}$ et Si qui présentent des rapports inférieurs à 1. Ces éléments sont peu corrélés aux autres éléments ou entre eux. Néanmoins ces trois éléments sont retenus par le couvert végétal sur les deux sites.

\section{DISCUSSION}

Le comportement de chaque élément trace peut s'interpréter en fonction de sa structure atomique, de ses propriétés chimiques propres et de son origine possible (Olive et Étienne, 1961).

$\mathrm{Rb}$ et $\mathrm{K}$, de même que $\mathrm{Ca}$ et $\mathrm{Sr}$, font partie de la même famille d'éléments (alcalins et alcalino-terreux respectivement) et présentent de plus des tailles très voisines. Ceci signifie qu'ils se comportent de manière analogue s'il sont placés dans des conditions chimiques identiques (Mahan, 1977 ; Wedepohl, 1979 ; Jacks et al, 1989). C'est effectivement ce que suggèrent les corrélations fortes - et indépendantes des stations - mesurées entre les concentrations de $\mathrm{K}$ et $\mathrm{Rb}$ d'une part, et $\mathrm{Ca}$ et $\mathrm{Sr}$ d'autre part. Ainsi les mécanismes invoqués quant à l'origine de l'enrechissement de $\mathrm{K}$ et $\mathrm{Ca}$ sont valables pour $\mathrm{Rb}$ et $\mathrm{Sr}$ respectivement. L'enrichissement en $\mathrm{K}$ et $\mathrm{Rb}$ provient pour l'essentiel de la récrétion, comme le suggèrent les très faibles teneurs dans les précipitations hors couvert, l'indépendance des concentrations hors et sous couvert, la prédominance des formes solubles ioniques de ces éléments dans les feuiles (Clément, 1989), ainsi que les calculs de bilan effec- 




Fig 2. Corrélations entre les concentrations des éléments dans les pluviolessivats de Jura et Lutry. Dans chaque cadre sont figurés les coefficients de corrélation ( $r$ ), pour Jura, puis Lutry, et enfin les deux sites réunis. 


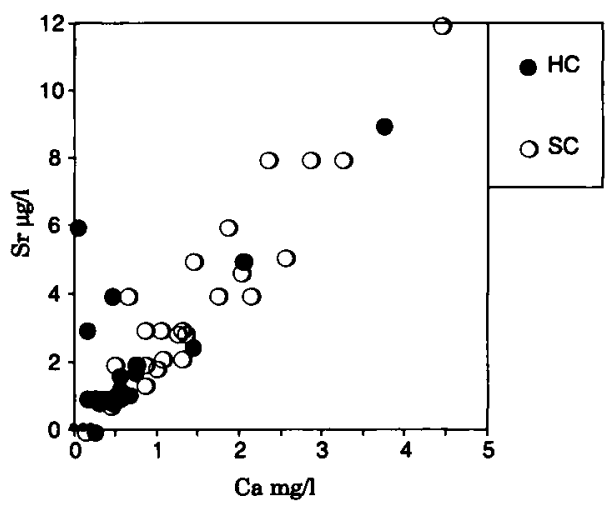

Fig 3. Corrélations entre $\mathrm{Ca}$ et $\mathrm{Sr}$ dans les solutions avant et après leur passage sous frondaison. HC: hors couvert; SC: sous couvert.
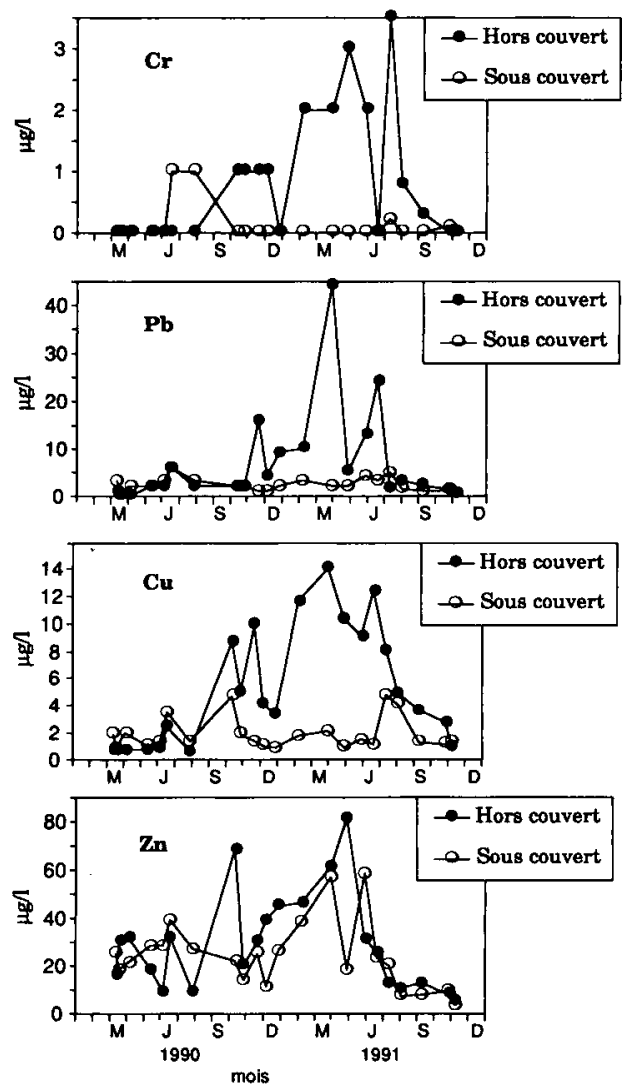

Fig 4. Évolution des concentrations de $\mathrm{Cd}, \mathrm{Pb}$, $\mathrm{Cu}$ et $\mathrm{Zn}$ hors et sous couvert à Jura. tués à l'échelle du sol (Atteia, 1992). Godt et al (1986) ont montré que la concentration de $\mathrm{K}$ augmente au cours du temps à la surface de rameaux protégés des dépôts secs. L'absence de corrélations avec d'autres éléments dans les précipitations sous couvert montre que cet enrichissement dépend peu de la composition chimique des solutions à la surface des feuilles. Cet enrichissement est beaucoup plus fort à Lutry qu'à Jura, ce que l'on peut mettre en relation avec le développement bien supérieur des houppiers dans le premier site, lié à la forte productivité de la forêt.

L'enrichissement en $\mathrm{Ca}$ est généralement attribué pour partie au dépôt sec et pour partie à la récrétion, cette dernière étant favorisée par l'acidité des dépôts, comme l'ont montré divers travaux expérimentaux (Potter, 1991 ; Scherbatskoy et Klein, 1983 ; Kaupenjohan et al, 1988 ; Leonardi et Flückiger, 1989). C'est aussi ce que suggèrent les corrélations entre $\mathrm{Ca}, \mathrm{Sr}$ et $\mathrm{SO}_{4}$ dans les pluviolessivats des deux stations. La contribution des dépôts secs est souvent estimée en appliquant I'hypothèse d'Ulrich (1983) développée par Bredemeier et al (1990) qui considèrent que les éléments sont déposés sur les rameaux selon les mêmes rapports de concentrations que dans les pluies, l'élément de référence étant $\mathrm{Na}$. Selon ce calcul, sur le site Jura, l'enrichissement de $\mathrm{Ca}$ serait entièrement dû au dépôt sec et de 3 $\mathrm{kg} / \mathrm{ha} / \mathrm{an}$. À Lutry le même calcul nous conduit à $12 \mathrm{~kg} / \mathrm{ha} / \mathrm{an}$ de dépôts secs alors que seuls $5,3 \mathrm{~kg}$ se retrouvent dans les pluviolessivats ; ainsi, 6 à $7 \mathrm{~kg} / \mathrm{ha} / \mathrm{an}$ seraient absorbés par la végétation.

Nous retrouvons le même scénario pour Sr car les 2 éléments sont corrélés en solution. Ces résultats sont contraires à ceux de Gosz et Moore (1989) et Grauschtein et Amstrong (1983), montrant que Sr est récrété par les conifères, et de Scherbatskoy et Klein (1983), prouvant l'existence de la récrétion de Ca. De plus, Grosch (1986) 
montre que, lorsque les tailles des particules de $\mathrm{Ca}$ et $\mathrm{Na}$ sont différentes, leurs vitesses de dépôt le sont aussi. Sur les deux sites, les apports de $\mathrm{Ca}$ et $\mathrm{Sr}$ dans les pluies sont indépendantes de ceux de $\mathrm{SO}_{4}$ alors que les droites de corrélation sont très nettes et de même pente sur les deux sites sous couvert. La végétation semble donc jouer un rôle important dans la régulation de ces concentrations. II semble en conséquence impossible, autant pour $\mathrm{Sr}$ que pour $\mathrm{Ca}$ de différencier la contribution des dépôts secs de celle de la récrétion en utilisant uniquement les relations entre composition des pluies et des pluviolessivats. L'origine de $\mathrm{Sr}$ dans les pluviolessivats est certainement mixte : dépôts secs, récrétion. Un raisonnement semblable peut être tenu pour $\mathrm{Mg}$.

$\mathrm{Mn}$ et $\mathrm{Ba}$ présentent des enrichissements différents à Lutry, sur sol acide, et à Jura sur sol calcaire. Cette particularité s'explique clairement en ce qui concerne $\mathrm{Mn}$ par les concentrations 100 à 200 fois plus élevées dans les solutions du sol de Lutry, comparées à celles de Jura (Atteia, 1992). On sait par ailleurs que les teneurs en $\mathrm{Mn}$ des aiguilles sont plus élevées en sol acide qu'en sol calcaire (Tyler et al, 1985) ; de même que $\mathrm{K}$ et $\mathrm{Rb}, \mathrm{Mn}$ est très peu présent dans les précipitations hors couvert et il se trouve majoritairement sous forme soluble dans les feuilles, de sorte que la récrétion est la principale source de l'enrichissement (Heinrichs et Mayer, 1980 ; Hofken, 1986 ; Godt et al, 1986). La relation nette entre concentrations de $\mathrm{Mn}$ et de $\mathrm{SO}_{4}$ dans les pluviolessivats suggère que l'intensité de la récrétion de cet élément est liée au niveau du dépôt acidifiant, contrairement à celle de $\mathrm{Rb}$ qui est indépendante de $\mathrm{SO}_{4}$. L'augmentation de l'enrichissement en $\mathrm{Ba}$ à "Jura" peut être rapprochée de la plus forte mobilité de $\mathrm{Ba}$ en milieu calcaire par rapport au milieu acide (Atteia, 1992). Nous pouvons ainsi appliquer à $\mathrm{Ba}$ le même raisonne- ment que pour Mn et conclure à l'existence de récrétion de $\mathrm{Ba}$ bien que ce phénomène soit moins net que pour $\mathrm{Mn}$.

En dehors d'une période de pollution spécifique à Jura, les métaux lourds présentent des degrés d'enrichissement de l'ordre.de 1. Durant la période d'augmentation des apports à Jura, une accumulation au moins temporaire de $\mathrm{Cu}$ et $\mathrm{Pb}$ s'est produite. La différence avec $\mathrm{Zn}$, qui s'est peu accumulé durant cette période, tient à la plus faible affinité de $\mathrm{Zn}$ pour la matière organique (Kabata-Pendias et Pendias, 1985). De plus, d'autres cas d'accumulation de $\mathrm{Pb}$ dans le feuillage ont été décrits (Bergkvist, 1987). Les variations de concentration des métaux lourds dans les pluviolessivats sont plus influencées par les concentrations de $\mathrm{SO}_{4}$ que par les variations de concentrations des métaux lourds dans les pluies. Ceci confirme l'existence d'un mécanisme de rétentionlibération des métaux lourds par le feuillage, commandé par la composition chimique des films d'eau à la surface du feuillage (Lindberg et al, 1982). On retrouve ici l'observation concernant $\mathrm{Ca}$ et $\mathrm{Sr}$.

Plusieurs auteurs citent des valeurs d'enrichissement importantes pour les métaux lourds dans des régions polluées : 2 à 8 pour $\mathrm{Cu}$ (Gietl et Rall, 1986 ; Brechtel et al, 1986), jusqu'à 30 pour Zn (Brechtel et al, 1986). De plus Godt et al (1986) montrent que les rameaux exposés à de forts niveaux de pollutions sont recouverts de dépôts importants de métaux lourds ( $\mathrm{Zn}$, $\mathrm{Cu}, \mathrm{Pb})$ par rapport à des témoins protégés. Nous avons signalé que les concentrations de métaux lourds dans les pluies sur les sites étudiés étaient faibles par rapport à d'autres régions d'Europe. La faible pollution des sites est à notre avis la raison du faible enrichissement des pluviolessivats en ces éléments. Turner et al (1985) ont d'ailleurs constaté dans un site peu pollué, un degré d'enrichissement similaire pour $\mathrm{Pb}$ à celui de cette étude. Seule une 
analyse complémentaire des surfaces foliaires permettrait de quantifier le dépôt sec de ces éléments.

Nous n'avons pas trouvé de facteur explicatif de la rétention de $\mathrm{Si}, \mathrm{B}$ et $\mathrm{Fe}$, si ce n'est leur capture sous forme particulaire (Si, Fe) par les cires des aiguilles (Rose, communication personnelle). Les données bibliographiques concernant des sites peu pollués manquent. On peut cependant remarquer que $\mathrm{Si}$ et $\mathrm{B}$, qui possèdent des structures chimiques semblables (Mahan, 1977) sont parallèlement retenus.

\section{CONCLUSION}

Dans des pessières peu polluées de Suisse romande, les dynamiques des éléments traces appellent les remarques suivantes.

i) L'enrichissement en Rb comme en K lors de la traversée du couvert est indépendant de celui des autres éléments. II exprime pour l'essentiel la récrétion.

ii) L'alimentation minérale de l'arbre influe sur l'enrichissement en $\mathrm{Mn}$ et $\mathrm{Ba}$. Cet enrichissement reflète ainsi les concentrations des solutions de sol.

iii) Une partie des flux de $\mathrm{Si}$, Fe et B sont interceptés par le feuillage, le mécanisme de cette rétention n'est pas élucidé.

iv) $\mathrm{V}, \mathrm{Cr}, \mathrm{Mn}, \mathrm{Ni}, \mathrm{Zn}, \mathrm{Sr}, \mathrm{Ba}$ ainsi que $\mathrm{Ca}$ et $\mathrm{Mg}$ sont déposés sous forme sèche et récrétés dans des proportions variables. Leurs concentrations instantanées dans les précipitations sous couvert semblent déterminées par les conditions chimiques à la surface des aiguilles de sorte qu'il est extrêment difficile de différencier la part atmosphérique de la part biologique. Les calculs effectués à partir de $\mathrm{Na}$ comme référence en ce qui concerne les dépôts secs ne peuvent dans ce cas être considérés comme fiables. Par contre un enrichissement expérimental du végétal en éléments présentant des analogies de structure atomique avec ceux dont on souhaite connaître l'origine pourrait être une voie prometteuse pour quantifier la récrétion.

\section{RÉFÉRENCES}

Aschan C, Dambrine E, Nourisson G, Tabeau M (1991) Dépôt d'éléments minéraux sur les écosystèmes naturels terrestres (INRA Nancy, ed) rapport au ministère de l'Environnement, $160 \mathrm{pp}$

Atteia $O$ (1992) Rôle du sol dans le transfert d'éléments traces en solution - Application à l'étude de quelques écosystèmes d'altitude. EPFL Lausanne, Thèse $n^{\circ} 1031,253 \mathrm{pp}$

Atteia $O$ (1993) Atmospheric input of major and trace elements in western Switzerland. Atmos Environ (sous presse)

Bergkvist B (1987) Soil solution chemistry and metal budgets of spruce forest ecosystems in Southern Sweden. Water Air Soil Poll 33, 131-154

Bergkvist B, Folfeson F, Berggren D (1989) Fluxes of $\mathrm{Cu}, \mathrm{Pb}, \mathrm{Cd}, \mathrm{Cr}$ and $\mathrm{Ni}$ in temperate forest ecosystems. Water Air Soil Poll 47, 217-286

Brechtel HM, Balazs A, Lehnardt F (1986) Precipitation input of inorganic chemicals in the open field and in forest stands - Results of investigations in the state of Hesse. In: Atmospheric pollutants in forest areas (HW Georgii, ed) Reidel, pp 47-68

Bredemeier M (1988) Forest canopy transformation of atmospheric deposition. Water Air Soil Poll 40, 121-138

Bredemeier M, Matzner E, Ulrich B (1990) Internal and external proton load to forest soils in northern Germany. J Env Qual 19, 469-477

Bresser AHM (1990) Acidic precipitation research in the Netherlands. In: Acidic Precipitation, International overview and assessment (AHM Bresser, W Salomoms, eds). Springer-Verlag, 159-182

Canter LW (1986) Acid rain and dry deposition, Lewis Publishers, Chelsea, Michigan, pp 370

Clement A (1989) Equilibre ionique du tissu foliaire de l'épicéa et du pin noir d'Autriche. Thèse université Nancy I. 382 pp 
Cronan CS, Reiners WA (1983) Canopy processing of acidic precipitation by coniferous and hardwood forest in New England. Oecologia 59, 216-223

Ellis VA, Verfaillie JR, Kummerow J (1983) Nutrient gain from wet and dry atmospheric deposition and rainfall acidity in the southern $\mathrm{Ca}$ lifornia Caparral. Oecologia 60, 1, 118-121

Galloway JN, Thornton JD, Norton SA, Volvhok AL, Mclean RA (1982) Trace metals in atmospheric deposition: a review and assessment. Atmos Environ 16, 1677-1700

Garland JA (1979) Dry deposition of gaseous pollutants AERE, Harwell, England

Garten CT, Bondietti EA, Lomax RD (1988) Contribution of foliar leaching and dry deposition to sulfate in net throughfall below deciduous trees. Atmos environ 22,7 1425-1432

Gietl G, Rall AM (1986) Bulk deposition into the catchment "Grosse Ohe": results of neighbouring sites in the open air and under spruce at different altitudes. In: Atmospheric pollutants in forest areas (HW Georgii, ed), Reidel, pp 263-274

Godt J, Schmidt M, Mayer R (1986) Processes in the canopy of trees: internal and external turn-over of elements. In: Atmospheric pollutants in forest areas (HW Georgii, ed) Reidel, pp 263-274

Gosz JR, Moore DI (1989) Strontium isotope studies of atmospheric inputs to forested watersheds in New Mexico. Biogeochemistry 8 , 155-134

Grauschtein WC, Amstrong RL (1983) The use of Sr87/Sr86 ratios to measure atmospheric transport into forested watersheds. Science 219, 289-292

Grennfelt P, Bengston C, Skaerby L (1978) Estimation of the atmospheric input of acidifying substances to a forest ecosystem. Swedish water and air pollution research laboratory, Goteborg, Sweden

Grosch S (1986) Wet and dry deposition of atmospheric trace elements in forest areas. In: Atmospheric pollutants in forest areas (HW Georgii, ed), D Reidel, pp: 35-46

Heinrichs H, Mayer R (1980) The role of forest vegetation in the biogeochemical cycle of heavy metals. J Envir Qual 9, 1, 111-118

Hennequin CL, Hervouet G, Brun C (1984). In; Livre blanc des pluies acides. Première approche scientifique du problème en France.
Secrétariat d'État à l'Environnement et à la Qualité de la vie. pp 145-160

Hoffmann WA, Lindberg SE, Turner RR (1980) Precipitation acidity: the role of the forest canopy in acid exchange. J Envir Qual 9, 95-100

Hofken KD (1986) Input of acidifiers and heavy metals to a german forest area due to dry and wet deposition. In: Effects of Accumulation of air pollutants in forest ecosystems (B Ulrich, $\mathrm{H}$ Pakrrath, eds)

Horntvedt R, Dollards GH, Joranger E (1980) Effects of acid precipitation on soil and forest: II Atmospheric-vegetation interactions. In: Ecological impact of acid precipitation (D Drablos, A Tollan, eds), SNSF pronect, Oslo, pp 192

Ibrahim M, Barrie L, Fanaki F (1983) Experimental and theoritical investigation of the dry deposition of particles to snow. Pine trees and artificial collectors. Atmos environ 17, 4, $1781-788$

Ivens W, Draaijers G, Bleuten W, Bos M (1989) The impact of air-borne ammonia from agricultural sources on fluxes of nitrogen and sulfur toward forest soils. Catena 16, 535-544

Jacks G, Aberg G, Hamilton J (1989) Calcium budgets for catchments as interpreted by strontium isotopes. Nord Hydrol 20, 85-96

Järvinen $O$ (1986) Papers of the national board of waters 408

Kabata-Pendias A, Pendias H (1985) Trace elements in soils and plants. CRC Press Inc, Boca Raton, Florida

Kaupenjohan M, Schneider BU, Hantschel R, Zech W, Horn R (1988) Sulphuric acid treatment of Picea abies : Effects on nutrient solution, throughfall chemistry and tree nutrition. Z Pflanzenernähr Bodenk 151, 123-126

Kostelnik KM, Lynch JA, Grimm JW, Corbett ES (1989) Sample size requirements for estimation of throughfall chemistry beneath a mixed hardwood forest. J Envir Qual 18, 274-280

Leonardi S, Flückiger W (1989) Effects of cation leaching on mineral cycling and transpiration: investigations with beech seedlings (Fagus sylvatica L). New Phytol 111, 173-179

Lindberg SE, Turner RR, Lovett GM (1982) Processes of atmospheric deposition of metals and acids to forests. Oak Ridge national laboratory, Oak Ridge, Tennessee

Mahan BH ( 1977$)$ Chimie Inter Editions, Paris, SA 
Marschner H (1986) Mineral Nutrition of higher plants. Academic Press Limited, San Diego, pp 674

Olive A, Étienne D (1961) Les terres rares. Ann Cosmochimie 3, 19-42

Potter CS (1991) Nutrient leaching from Acer rubrum leaves by experimental acid rainfall. Can J For Res 21, 222-229

Scherbatskoy T, Klein RM (1983) Response of spruce and birch foliage to leaching by acidic mists. J Envir Qual 12, 189-195

Smidt S (1988) Messungen der nassen deposition in Osterreich. Messtellen, Jahresmesseregebnisse, Literatur FBVA-Berichte 27

Summers PW, Bowersox VC, Stenslet GJ (1986) The geographical distribution and temporal variations of acidic deposition in eastern North America. Water Air Soil Poll 31, 523-535

Swank WT (1984) Atmospheric contribution to forest nutrient cycling. Water Resources Bulltin 20(3), 313-321
Tukey JB, Wittwer SH (1957) Loss of nutrients by foliar leaching as determined by radioisotopes. J Amer Soc Hort Sci 71, 496-506

Turner RS, Johnson AH, Wang D (1985) Biogeochemistry of lead in McDonalds Branch Watershed, New Jersey pine barrens. J Envir Qual 14, 305-313

Tyler G, Steines E, Folkeson L, Lobersli E, Nyholm E (1985) Research report, Lund University, Dept of Plant Ecology, Lund, Sweden

Ulrich B (1983) Interaction of forest canopies with atmospheric constituants : $\mathrm{SO}_{2}$, alkali and earth alkali cations and chloride. In: "Effects of accumulation of air pollutants in forest ecosystems", Reidel Publ, Dordrecht, Holland, 33-45

Veen AL, Dolman AJ (1989) Water dynamics of forests, one-dimensional modelling. Prog Physic Geography 13, 471-506

Wedepohi KH (1968-1979) Handbook of geochemistry. Springer Verlag, Berlin 